\title{
2 Vom Stethoskop zur Blockchain - Technologische Disruption im Gesundheitswesen
}

\author{
Sebastian Krolop
}

\section{Schumpeter und die schöpferische Kraft der Zerstörung im Gesundheitswesen}

Vor nicht einmal einem Jahr schrieb ich das Kapitel „Digitalisierung und Systempartnerschaft - was Schumpeter darüber denken würde“ (Krolop 2016) für ein Buchprojekt. Als ich zur Vorbereitung für diesen Beitrag mein „altes“ Werk durchsah musste ich schmunzeln. Tatsächlich lässt sich feststellen, dass ich meine knapp zehn Monate alte Einschätzung zur Auswirkung der Digitalisierung auf bestehende Geschäftsmodelle schlicht und einfach unterschätzt habe. Joseph Schumpeter hingegen würde sich durch die zu beobachtende schöpferische Kraft der Zerstörung wahrlich bestätigt fühlen.

Diese kreative Zerstörung erleben wir heute über fast alle Industrien hinweg. Die Digitalisierung treibt die Transformation der Weltwirtschaft in einem atemberaubenden Tempo voran (Krolop 2014). Ob Medien, Touristik, Banking oder Handel durch die intelligente Verknüpfung von Daten entstehen neue Angebote, die oft einen neuen und intuitiven Mehrwert für Kunden generieren und somit etablierte Anbieter verschwinden lassen. Das Internet der Dinge und Industrie 4.o heben weiteres Potenzial durch die Verknüpfung von Maschinen und die kontinuierliche Bewertung von Informationen. Die Entwicklung von „artificial intelligence“ über „machine learning“ und „deep learning“ hin zu „cognitive learning“ durch neuronale Netzwerke haben einen Reifegrad erreicht, welche Parallelen zum menschlichen Gehirn zulassen. 
Es entwickeln sich neue Anbieter, die durch kluge und intuitive Lösungen das Vertrauen der Verbraucher gewinnen. Diese digitalen Ceschäftsmodelle bilden geschlossene Ökosysteme und lassen dabei etablierte und traditionelle Anbieter außen vor. Die Unternehmen Airbnb und Uber sind aktuelle Beispiele dafür, wie kundenzentrierte digitale Plattformen klassische Geschäftsmodelle wie Hotellerie oder Taxigewerbe zum Wanken bringen können. Die 2008 gegründete Plattform Airbnb, welche Privatleuten die Vermietung ihrer Wohnungen und Zimmer vermakelt, wird mit über 30 Mrd. Dollar, dass erst 2009 gegründete Start-up Uber wird als Fahrdienst-Vermittler zeitweise auf über 6o Mrd. Dollar bewertet. Uber ist demnach mehr wert als die DAX-Unternehmen Lufthansa, ProSiebenSatı Media, RWE, Commerzbank und thyssenkrupp - zusammen.

Allerdings haben in der letzten Zeit einige Skandale einen Schatten auf das Unternehmen und dessen Bewertung geworfen. Wer nun glaubt, dass das Phänomen Uber ein überschätzter, sich selbstregulierender Hype sei, sollte einen Blick ins Reich der Mitte wagen. Dort konnte der Uber Konkurrent Didi mit einem identischen Geschäftsmodell nicht nur Apple zu einer Investition von ca. 1 Mrd. Dollar bewegen, sondern wird auch auf ca. 50 Mrd. Dollar bewertet.

\section{Where Do Firms Go When They Die?}

Eine Studie der Yale School of Management (Foster 2015) veranschaulicht, wie sich die Mortalitätsrate von Unternehmen in den letzten 100 Jahren entwickelt hat. Sie analysiert die Aktien der 500 größten US-Konzerne und stellt fest, dass Unternehmen vor 100 Jahren im Schnitt 67 Jahre alt wurden. 2015 wurden sie im Schnitt 15 Jahre alt. Alle zwei Wochen verschwindet ein S\&P-50o-Konzern vom Markt, bis 2027 sollen drei Viertel der Top 500 durch neue ersetzt werden.

Diese rasanten Veränderungen lassen sich auch in einer Analyse der teuersten zehn Unternehmen der USA nachvollziehen. Aus den Top-Zehn Unternehmen im Jahre 2007 finden sich 2017 nur drei in dieser Liste wieder. Das wertvollste Unternehmen in den USA im Jahre 2007 war Exxon Mobile mit einem Börsenwert von über 330 Mrd. Euro. Zehn Jahre später liegt Exxon nur noch auf Platz acht - mit einem niedrigeren Börsenwert von ca. 310 Mrd. Euro. Die aktuelle Nummer eins, Apple (Börsenwert ca. 670 Mrd. Euro), und zwei, Alphabet/Google (Börsenwert ca. 520 Mrd. Euro), sind Neulinge in dieser Wertung. Die alte und neue Nummer drei ist Microsoft. Microsoft konnte innerhalb von zehn Jahren seinen Marktwert von ca. 220 Mrd. Euro auf über 460 Mrd. Euro mehr als verdoppeln. Facebook und Amazon auf Platz fünf und sechs runden die Liste der digitalen Neulinge ab.

Bedenklich invers zeichnet sich die Situation bei den zehn größten Unternehmen in Deutschland ab. Sieben Unternehmen finden sich sowohl 2007 als auch 2017 wieder. Leider ist dies nicht unbedingt als Stärke der deutschen Industrie zu werten, da die Entwicklung der Börsenwerte signifikant unter der der amerikanischen Konkurrenten liegt. Allein der „Neuling“ Apple ist so viel wert wie die acht wertvollsten deutschen Unternehmen zusammen. Nur SAP konnte als ehemalige Nummer sechs und aktuelle Nummer eins seinen Börsenwert von ca. 51 Mrd. Euro auf 111 Mrd. Euro annähernd verdoppeln. Des Weiteren ist SAP das einzige digitale Technologie-Unternehmen in der deutschen Liste, die restlichen Unternehmen stammen aus klassischen Industrien wie Chemie, Automobil oder Versicherung. 


\section{Digitalfreie Zone deutsches Gesundheitssystem?}

Während sich also die Transformation der Weltwirtschaft im vollen Gange befindet scheinen das deutsche Gesundheitssystem und die deutsche Krankenhauslandschaft aus Sicht der Digitalisierung eher im letzten Jahrtausend stecken geblieben zu sein. Der international anerkannte Score „Electronic Medical Record Adoption Model; EMRAM“ der HIMSS ist ein Acht-Stufen-Modell, welches den digitalen Reifegrad von Krankenhäusern misst und vergleichbar macht. Vereinfacht ausgedrückt bedeutet die Stufe Null, dass keine digitalen Informationssysteme angewandt werden, die höchste Stufe Sieben bedeutet ein papierloses Krankenhaus. Ein Europavergleich der HIMSS aus dem Jahr 2017 ermittelt einen Mittelwert der untersuchten Krankenhäuser in Dänemark von 5,3, in den Niederlanden von 4,8 und als Schlusslicht Deutschland mit einem Wert von nur 2,2.

Hierfür mag es viele Gründe geben. Ein Treiber ist sicherlich der fehlende finanzielle Freiraum für innovative Investitionen im deutschen Gesundheitswesen. In unserem „Krankenhaus Rating Report 2016: Mit Rückenwind in die Zukunft?“ (Krolop 2016) zeigen wir auf, dass jedes neunte Krankenhaus von einer Insolvenz bedroht ist und fast jedes zweite Krankenhaus nicht ausreichend investieren kann. Wir identifizieren einen Investitionsstau von stolzen 27,8 Mrd. Euro. Dieses Geld fehlt, um strukturelle und digitale Änderungen voranzutreiben.

In der Vergangenheit konnte man bei den Krankenhäusern, Ärzten, Krankenkassen usw. neben diesen quantifizierbaren finanziellen Restriktionen allerdings auch deutliche Ressentiments gegenüber Transparenz und Vergleichbarkeit durch Digitalisierung spüren. Jahrelang wurden Initiativen wie die Einführung der elektronischen Gesundheitskarte durch zahlreiche Bedenken verzögert und teilweise torpediert - eine Entwicklung, die glücklicherweise in den letzten Jahren rückläufig ist. Nun stellt sich die Frage, wie gefährlich diese fehlende Offenheit gegenüber digitalen Innovationen für die Teilnehmer des deutschen Gesundheitssystems ist. So bahnen sich insbesondere im Gesundheitswesen technologische Entwicklungen an, die das Potenzial der schöpferischen Kraft der Zerstörung aufweisen. Den größten disruptiven Einfluss haben dabei die Digitalisierung der Medizin, die personalisierte Medizin und der informierte und mündige Patient.

Mit enormer Geschwindigkeit entstehen neue technologische Plattformen, welche das Potenzial der personalisierten Medizin heben können. Durch die Sequenzierung des menschlichen Genoms können wir unser Erbgut entschlüsseln und kausale Zusammenhänge zwischen Genvarianten, molekularbiologischen Konstellationen und Krankheitsprozessen des individuellen Patienten berücksichtigen. Zusammen mit der Möglichkeit der automatischen Erfassung und Befundung von Sensorik-Daten, u.a. Wearables, werden wir in die Lage versetzt, Diagnostik und Therapie individuell zu verfeinern und dadurch ungewünschte Nebeneffekte und Komplikationen zu vermindern oder gar zu vermeiden.

\section{Disruption durch den mündigen Patienten}

Der größte Katalysator der Veränderungen ist aber als treibende Kraft der informierte und mündige Patient. Trotz der enormen Kosten des Gesundheitssystems beschleicht ihn das unangenehme Gefühl, dass seine Behandlung qualitativ nicht im- 
mer optimal verläuft. Er erkennt auch, dass medizinische Informationen, die früher nur für Ärzte zugänglich waren, nun digital vorliegen und durch weitere Informationen aus dem sozialen Netzwerk der Patienten sogar mit zusätzlichem Mehrwert angereichert sind. Diese neue Transparenz führt dazu, dass der einfache Patient zum informierten und mündigen Klienten wird, welcher zunehmend Verantwortung für die eigene Gesundheit übernimmt.

Zugegebenermaßen ist die bisherige Architektur des Internets als Rückgrat für Digitalisierung im Gesundheitswesen aufgrund von Sicherheitsaspekten alles andere als optimal. Man kann als Individuum dem Internet nicht trauen, daher nutzen wir (vertrauensvolle) intermediäre Dritte in Form von Banken, Email-, Reise- oder Einkaufplattformen. Für Patienten stellt diese potenzielle Unsicherheit des Internets eine - zumindest subjektiv - höhere Hürde dar. Der oft zitierte Ausdruck „Datenschutz ist etwas für Gesunde“ spiegelt dieses Dilemma wider: als Gesunder steht die Datensicherheit im Vordergrund. Je kranker der Patient ist, desto höher ist sein Leidensdruck und die Hoffnung oder Gewissheit, dass durch den elektronischen Datenaustausch Klarheit, Linderung oder gar Heilung möglich ist. Somit steigt die Compliance von chronisch Kranken gegenüber diesen modernen Medien.

Was wäre nun, wenn es eine Möglichkeit gäbe, diesen Grundfehler des Internetprotokolls zu beheben? Was wäre, wenn es eine Möglichkeit gäbe, die offensichtlichen Vorteile der Digitalisierung und Vernetzung nicht nur absolut sicher, sondern auch zu 100\% vertrauensvoll, transparent und nachvollziehbar zu gestalten? Diese unglaubliche Technologie basiert auf sogenannten Blockchains und Smart Contracts (Giese et al. 2016). Vereinfacht dargestellt basieren Blockchains auf einer dezentralen Datenarchitektur („Distributed Ledger Technology“), welche sich durch Sicherheit, Verfügbarkeit und eben einer Dezentralität auszeichnet, welche ein Hacken der Architektur quasi unmöglich macht. Experten sehen die Blockchain-Technologie als die größte Erfindung - seit dem Internet.

Eine Technologie, die auf Vertraulichkeit, Authentizität und Integrität basiert wäre ein wahrer Segen für Banking oder Supply-Chain-Management. Für das Cesundheitswesen wäre es jedoch der Urknall, eine Tsunami-artige Disruption ungeahnten Ausmaßes. Die bisherige Asymmetrie von Wissen zwischen Gesundheitskonsumenten oder Patienten und Experten des Gesundheitswesens wäre auf einen Schlag aufgehoben. Diagnostik und Therapie würde nicht mehr auf Eminenz sondern auf Evidenz basieren. Unzählige Intermediäre würden über Nacht nicht mehr gebraucht werden und verschwinden.

Nun lässt sich spekulieren, wie in zehn Jahren die Liste der zehn wertvollsten Unternehmen der USA aussehen wird. Ganz nach Schumpeter gehe ich die Wette ein: die Mehrheit der Unternehmen waren 2017 nicht auf der Liste, mindestens zwei Unternehmen kommen aus dem Bereich „Digitale Gesundheitsorganisation“, beide Unternehmen besitzen keine eigenen Krankenhausbetten.

\section{Literatur}

Augurzky B, Krolop S, Pilny A, Schmidt C, Wuckel C (2016) Krankenhaus Rating Report 2016 - Mit Rückenwind in die Zukunft? medhochzwei Verlag

Foster (2015) Where Do Firms Go When They Die. The Atlantic. Stand: 12. April 2015. URL: https://www.theatlantic.com/business/archive/2015/04/where-do-firms-go-when-they-die/390249/ (abgerufen am 21.08.2017) 
Giese P, Kops M, Wagenknecht S, de Boer D, Preuss M (2016) Die Blockchain Bibel: DNA einer revolutionären Technologie, BTC-ECHO

Krolop S (2014) Kreative Zerstörung. In: Ampere 4/2014, S. 14. URL: https://www.zvei.org/fileadmin/user_upload/Presse_und_Medien/Publikationen/AMPERE/2014/AMPERE_4.2014__Medizintechnik/Ampere-42014-Medizintechnik.pdf (abgerufen am 21.08.2017)

Krolop S (2016) Digitalisierung und Systempartnerschaft - was Schumpeter darüber denken würde. In: Lohmann H, Kehrein I, Rippmann K (Hrsg.) Markenmedizin für informierte Patienten: Strukturierte Behandlungsabläufe auf digitalem Workflow. S. 142-148. medhochzwei Verlag 